\title{
Holographic superconductor with disorder
}

\author{
D. Areán, ${ }^{1,2, *}$ A. Farahi, ${ }^{3, \dagger}$ L. A. Pando Zayas, ${ }^{3, \$}$ I. Salazar Landea, ${ }^{4,1, \S}$ and A. Scardicchio ${ }^{1,2, \uparrow}$ \\ ${ }^{1}$ International Centre for Theoretical Physics (ICTP), Strada Costiera 11, I 34014 Trieste, Italy \\ ${ }^{2}$ INFN, Sezione di Trieste, Strada Costiera 11, I 34014 Trieste, Italy \\ ${ }^{3}$ Michigan Center for Theoretical Physics, University of Michigan, Ann Arbor, Michigan 48109, USA \\ ${ }^{4}$ Instituto de Física La Plata (IFLP) and Departamento de Física, Universidad Nacional de La Plata, \\ CC 67, 1900 La Plata, Argentina
}

(Received 30 October 2013; published 13 May 2014)

\begin{abstract}
We study the effects of disorder on a holographic superconductor by introducing a random chemical potential on the boundary. We consider various realizations of disorder and find that the critical temperature for superconductivity is enhanced. We also present evidence for a precise form of renormalization in this system. Namely, when the random chemical potential is characterized by a Fourier spectrum of the form $k^{-2 \alpha}$ we find that the spectra of the condensate and the charge density are again power laws, whose exponents are accurately and universally governed by linear functions of $\alpha$.
\end{abstract}

\section{INTRODUCTION}

Disorder is a fundamental paradigm in condensed matter physics as it provides a crucial step away from clean systems towards realistic ones. One of the most striking and ubiquitous manifestations of disorder in noninteracting quantum systems is the phenomenon of Anderson localization [1], where the conductivity can be completely suppressed by quantum effects. Due to the technical difficulties involved, the study of the interplay between disorder and interactions in quantum systems has seen little progress on the theoretical side. Recently however, in the context of disordered conductors, Basko, Aleiner and Altshuler took a formidable step forward by presenting compelling evidence in favor of a many-body localized phase, based on an analysis of the perturbation theory in electron-electron interaction to all orders [2]. Subsequent works (see [3-7] and references therein) have confirmed and sharpened the picture of the existence of a phase transition separating the weak and strong interacting limit of electrons in disordered potentials.

The AdS/CFT correspondence provides a natural framework where some strongly coupled field theory systems can be described in terms of weakly coupled gravity. It is only natural to try to understand the interplay of disorder and strong interactions in this context. Indeed, there has been a number of discussions along these lines [8-14]. In this paper, however, we follow a direct approach of coupling a given operator to a randomly distributed space-dependent source. We essentially translate a typical condensed matter protocol into the AdS/CFT framework.

\footnotetext{
*darean@ictp.it

† aryaf@umich.edu

t1pandoz@umich.edu

\$peznacho@gmail.com

ascardic@ictp.it
}

One particularly important application of disorder is in the context of dirty superconductors which have a rich history in condensed matter physics dating back to the pioneering work of Anderson in 1959 [15]. For many years Anderson's theorem, stating that superconductivity is insensitive to perturbations that do not destroy timereversal invariance (pair breaking), provided the central intuition. Critiques to Anderson's argument were raised, for example, in [16-18] where the effects of strong localization were considered. More generally, the question of the role of interactions, in particular, the Coulomb interaction in dirty superconductors cannot be considered settled. In view of this situation, it makes sense to consider alternative models where the problem can be analyzed in full detail.

The AdS/CFT correspondence has succeeded in constructing a holographic version of superconductors $[19,20]$, for comprehensive reviews see $[21,22]$. Thus, the AdS/CFT correspondence provides a perfect playground to explore the role of disorder within a model for strongly interacting superconductivity. This is precisely what we do in this manuscript by promoting the chemical potential in the holographic superconductor to a random space-dependent function. The main rationale for this choice of disorder relies on the fact that the chemical potential defines the local energy of a charged carrier placed at a given position $x$ coupling with the particle number $n(x)$ locally. Therefore, our choice of disorder replicates a local disorder in the onsite energy. This is the simplest protocol one would implement. Moreover, once disorder is introduced in such an interacting system, all observables will become disordered and, therefore, the physics is not expected to depend on the way disorder is implemented.

In this paper we focus on two aspects. The first one is the effect of disorder on the critical temperature for superconductivity: we find that the critical temperature for setting the superconductivity is increased by the presence 


\section{AREÁN et al.}

of the disorder. The second aspect is the study of certain universality of the power spectra of the condensate and charge density as functions of the power spectrum of the signal defining the noise. Namely, for a given random signal with power spectrum of the form $k^{-2 \alpha}$ we study the power spectrum of the condensate $k^{-2 \Delta(\alpha)}$ and of the charge density $k^{-2 \Gamma(\alpha)}$ and report some interesting universal behavior. We interpret this behavior as a particular form of renormalization of small wavelengths.

\section{NOISY HOLOGRAPHIC SUPERCONDUCTOR}

To build a noisy holographic $s$-wave superconductor in $2+1$ dimensions we start with the action introduced originally in $[19,20]$. Namely, we consider the dynamics of a Maxwell field and a charged scalar in a fixed metric background:

$S=\int d^{4} x \sqrt{-g}\left(-\frac{1}{4} F_{\mu \nu} F^{\mu \nu}-\left(D_{\mu} \Psi\right)\left(D^{\mu} \Psi\right)^{\dagger}-m^{2} \Psi^{\dagger} \Psi\right)$.

The system is studied on the Schwarzschild-AdS metric:

$$
\begin{aligned}
d s^{2} & =\frac{1}{z^{2}}\left(-f(z) d t^{2}+\frac{d z^{2}}{f(z)}+d x^{2}+d y^{2}\right), \\
f(z) & =1-z^{3},
\end{aligned}
$$

where we have set the radius of $\operatorname{AdS}, R=1$, and the horizon at $z_{h}=1$. Let us now take the following (consistent) Ansatz for the matter fields:

$$
\begin{gathered}
\Psi(x, z)=\psi(x, z), \quad \psi(x, z) \in \mathbb{R}, \\
A=\phi(x, z) d t .
\end{gathered}
$$

The resulting equations of motion read

$$
\begin{gathered}
\partial_{z}^{2} \phi+\frac{1}{f} \partial_{x}^{2} \phi-\frac{2 \psi^{2}}{z^{2} f} \phi=0, \\
\partial_{z}^{2} \psi+\frac{1}{f} \partial_{x}^{2} \psi+\left(\frac{f^{\prime}}{f}-\frac{2}{z}\right) \partial_{z} \psi+\frac{1}{f^{2}}\left(\phi^{2}-\frac{m^{2} f}{z^{2}}\right) \psi=0 .
\end{gathered}
$$

In what follows we will choose the scalar to have $m^{2}=-2$, corresponding to a dual operator of dimension 2.

We shall first study the UV asymptotics of Eqs. (5) and (6). Near $z=0$ their solution is given by

$$
\begin{gathered}
\phi(x, z)=\mu(x)+\rho(x) z+\phi^{(2)}(x) z^{2}+o\left(z^{3}\right), \\
\psi(x, z)=\psi^{(1)}(x) z+\psi^{(2)}(x) z^{2}+o\left(z^{3}\right),
\end{gathered}
$$

PHYSICAL REVIEW D 89, 106003 (2014)

where $\mu(x)$ and $\rho(x)$ correspond to space-dependent chemical potential and charge density respectively. The functions $\psi^{(1)}(x)$ and $\psi^{(2)}(x)$ are identified, under the duality, with the source and vacuum expectation value of an operator of dimension 2. Subsequent $x$-dependent coefficients in this small $z$ asymptotic expansion are determined in terms of $\mu(x), \rho(x), \psi^{(1)}(x)$ and $\psi^{(2)}(x)$. In the IR, requiring regularity implies that $A_{t}$ vanishes at the horizon. Hence, we consider an asymptotic expansion of the form

$\phi(x, z)=(1-z) \phi_{h}^{(1)}(x)+(1-z)^{2} \phi_{h}^{(2)}(x)+\cdots$,

$\psi(x, z)=\psi_{h}^{(0)}(x)+(1-z) \psi_{h}^{(1)}(x)+(1-z)^{2} \psi_{h}^{(2)}(x)+\cdots$,

where the ellipses stands for higher order terms.

By redefining the scalar as

$$
\chi(x, z)=\frac{1-z}{z} \psi(x, z),
$$

we arrive at the following boundary value problem:

$\chi(x, 0)=0, \quad \phi(x, 0)=\mu(x), \quad \mathrm{UV} z \rightarrow 0$,

$\chi(x, 1)=0, \quad \phi(x, 1)=0, \quad$ IR $z \rightarrow 1$.

This choice of boundary conditions corresponds to spontaneous breaking of the $U(1)$ symmetry with order parameter $\langle\mathcal{O}\rangle \propto \psi^{(2)}(x)$. From now on we use the angle brackets associated with $\mathcal{O}$ exclusively to refer to average over $x$.

\section{INTRODUCING DISORDER}

We are interested in solving the system given by Eqs. (5) and (6) in the presence of disorder. Let us take the following form for the noisy chemical potential:

$$
\begin{aligned}
\mu(x) & =\mu_{0}+\epsilon \sum_{k=k_{0}}^{k_{*}} \sqrt{S_{k}} \cos \left(k x+\delta_{k}\right) \\
& =\mu_{0}+\epsilon \sum_{k=k_{0}}^{k_{*}} \frac{1}{k^{\alpha}} \cos \left(k x+\delta_{k}\right),
\end{aligned}
$$

where $S_{k}$ is the power spectrum and $\delta_{k} \in[0,2 \pi]$ are random phases. Ensemble averages means averaging over these phases. Unless stated otherwise, we consider $\alpha=1$. This means that our noise will be continuous but without welldefined derivatives in the limit $k^{*} \rightarrow \infty$. The correlation function of the noise is

$$
\left\langle\mu\left(x^{\prime}\right) \mu(x)\right\rangle-\mu_{0}^{2}=\sum_{k=k_{0}}^{k *} \frac{1}{k^{2}} \cos \left(k\left(x^{\prime}-x\right)\right) .
$$


We observe then that, in the limit $k^{*} \rightarrow \infty, k_{0}$ defines the inverse of the correlation length.

We discretize the space, and impose periodic boundary conditions in the $x$ direction, leading to $k$ with values:

$$
k_{n}=\frac{2 \pi n}{L} \quad \text { with } \quad 1 \leq n \leq \frac{L}{a}-1
$$

where $L$ is the length in the $x$ direction of our cylindrical space, and $a$ is the lattice spacing in $x$. Note that there is an IR scale given by $k_{0}$ and a UV scale defined by $k_{*}=\frac{2 \pi}{a}$. In the limit of a large number of modes, the form of Eq. (11) tends to a Gaussian distributed random function; also note that the power of $1 / k$ determines the differentiability properties of $\mu(x)$. We also considered other realizations of disorder, for example,

$$
\mu(x)=\mu_{0}+\epsilon(x),
$$

where $\epsilon(x)$ is a random function in $(-W, W)$ taking values at different lattice sites (with $W \leq \mu_{0}$ ). This is the extreme limit in which correlation is lost at a lattice site distance.

We have solved the original system of partial differential Eqs. (5) and (6) with boundary conditions (10) using finite difference with a second order central scheme. Most of the simulations were done independently in MathEMATICA and in Python. The latter ones ran in the University of Michigan Flux cluster. Our typical result contains a grid of $100 \times 100$ points but we have gone up to $200 \times 200$ to control issues of convergence and optimization. We used a relaxation algorithm to search for the solution and use an $\mathcal{L}_{2}$ measure for convergence which in most cases reached $10^{-16}$. As the source of randomness we used $\mu(x)$ given by (11) and also (14) for uniform and Gaussian distributions.

The scales involved in the problem are: AdS radius $R$, black hole temperature (horizon position) $z_{h}$ (set to 1 ), chemical potential $\mu_{0}$ and noise scale $k_{0}$. There is a distinction between the noise scale and the strength of the noise [variance of $\mu(x)$ ] which we parametrized by $\epsilon$. In the realization (11) we have $k_{0}$ and $\epsilon$. In the context of the realization (14) when $\epsilon(x)$ takes values in a uniform distribution in a certain interval, the discretization of the problem leads to the introduction of a cell in the grid, say of length $a$ along $x$. In this case assigning random values to $\mu(x)$ essentially amounts to introducing a correlation length, equal to $a$, such that inside this length the chemical potential is correlated but outside this length it is not. Note, however, that the IR scale in this choice continues to be $L$ which is related to $k_{0}$ above and the UV scale is $a$ which is related to $k_{*}$ above. For the realization of the noise following Eq. (11) we characterized the strength of disorder by introducing $w$ as $\epsilon=\frac{2}{5} \mu_{0}(w / 10)$, where $w=0$ corresponds to the homogeneous case and $w=10$ is the largest $\epsilon_{\max }=\frac{2}{5} \mu_{0}$. We choose this maximum value of the strength by demanding that $\mu(x)$ remains positive. This means that our analysis is restricted to a relatively small window of disorder. It might happen that as for some models of strongly coupled superfluids $[23,24]$ the superconducting phase is destroyed for large enough disorder.

For the noise realization given by Eq. (14), the maximum value $\epsilon_{\max }$ is $\mu_{0}$ and the dimensionless measure of the strength of disorder is proportional to $W / \mu_{0}$. Our definition of $w$ corresponds, in the standard solid state notation, to $1 / k_{F} l$, where $k_{F}$ is the Fermi momentum and $l$ is the meanfree path $[25,26]$. With this choice of scales we have essentially two dimensionless quantities $T / \mu_{0}$ and the strength of the noise $w$. The results we present below are most marked for the realization given by Eq. (11) but realizations in Eq. (14) show similar behavior.

\section{RESULTS}

An intuitive way to summarize our results is presented in Fig. 1 where we track the normalized value of the condensate $\langle\mathcal{O}\rangle / \mu^{2}$ as a function of the dimensionless strength of disorder, $w$. Here $\langle\cdots\rangle$ stands for average in $x$. A key feature of this diagram is that increasing disorder leads to a nonzero condensate in situations where the homogeneous case does not condense. The plot in Fig. 1 was made considering one realization of the noise. We have performed a preliminary analysis of the variance produced by the intrinsic randomness of the functions, namely, we have in some cases considered many realizations of the same noise $\left(\mu_{0}, k_{0}, k_{*}\right)$ in Eq. (11). We have found that up to $\mu_{0}$ around 4.00 this effect is negligible, however for smaller values of $\mu_{0}$ and with increasing values of the noise strength, $w$, the error becomes significant. This enhancement of superconductivity is one of our main results.

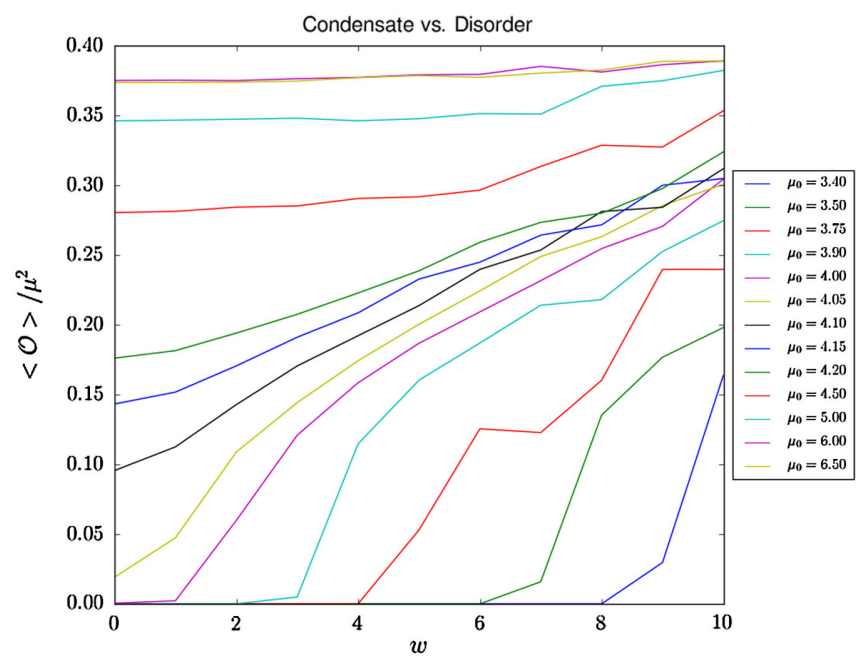

FIG. 1 (color online). Average of the condensate as a function of the strength of disorder using $k_{0}=1$. The value of the condensate grows with increasing disorder strength, $w$. 


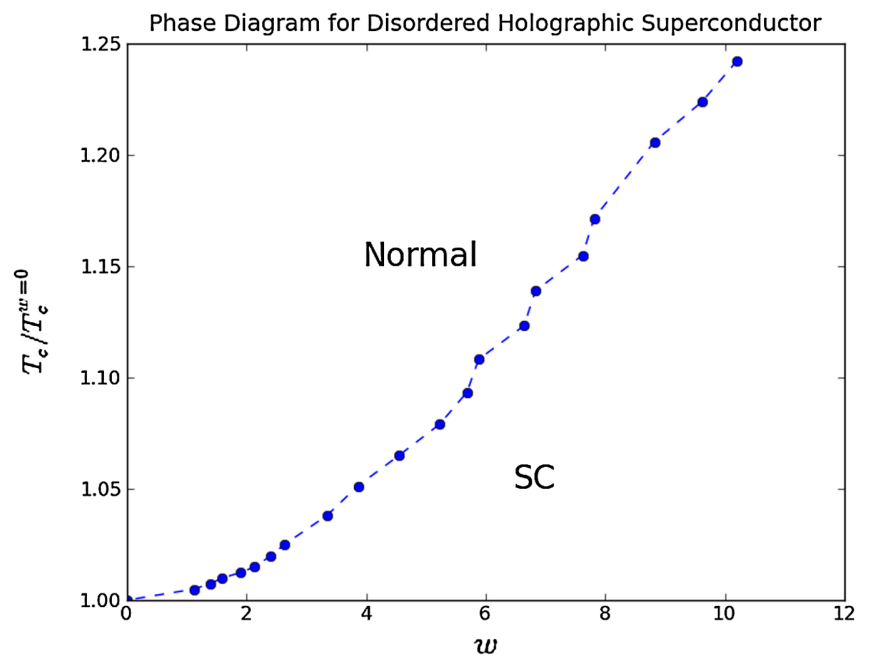

FIG. 2 (color online). Phase diagram, dependence of the critical temperature on the strength of the noise.

In a separate plot we explore, in more detail, the change of critical temperature $T_{c}$ with the strength of the noise $w$. Namely, we set a value ( $25 \%$ of the maximum) for which we consider the condensate to be clearly nonzero and find the smallest value of the homogeneous chemical potential for which this value of disorder $w$ leads to a nonzero condensate. The results are plotted in Fig. 2 which represents our version of the phase diagram of disordered superconductors as presented, for example, in [26] for realistic $s$-wave superconductors. We will briefly compare our results with the literature in the conclusions.

We note that the parameter $k_{0}$ affects the results numerically. Recall that the scale $k_{0}$ is related to the compactification length of the $x$ coordinate. What ultimately matters is the value of this length $L$ with respect to the range in the $z$ coordinate which is fixed to one. The simulations used to generate Figs. 1 and 2 used $k_{0}=1$. For example, taking $k_{0}=2 \pi$ leads to a more modest increment in the value of the condensate. Similarly, considering noise realizations of the form given in Eq. (14) leads to qualitatively similar results but the numerical enhancement is more modest.

One result that is rather generic is that for a highly irregular chemical potential, we find a very smooth dependence of the condensate on the coordinate $x$. A typical form of $\mu(x)$ and its corresponding $\mathcal{O}(x)$ are represented in Fig. 3.
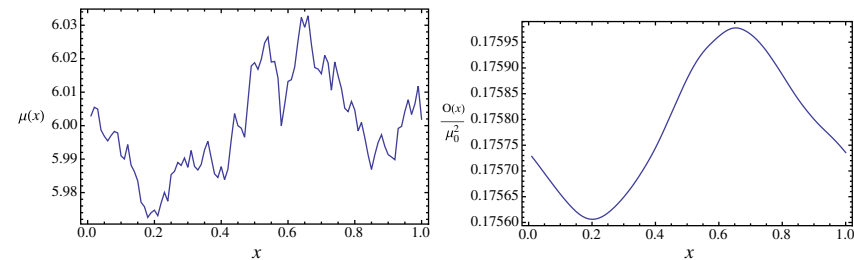

FIG. 3 (color online). Initial chemical potential profile $\mu(x)=$ $6.0+0.1 \sum_{n=1}^{100} \frac{1}{2 \pi n} \cos \left(2 \pi n x+\delta_{n}\right)$ (left panel) and the corresponding condensate profile (right panel).
On the other hand, the opposite happens for the charge density. A noisy chemical potential will translate into an even noisier charge density. A typical form of $\mu(x)$ and its corresponding $\rho(x)$ are represented in Fig. 4.

This kind of smoothing/roughening points to a renormalization of sorts, where higher harmonics in $\mathcal{O}$ are suppressed with respect to their spectral weight in $\mu$. Here we pursue this idea further. To characterize this renormalization quantitatively we consider a boundary chemical potential of the form presented in Eq. (11) but considering now different values for $\alpha$. The choice of this parameter $\alpha$ determines the degree of differentiability (smoothness) of the initial profile. To make the concept of renormalization more precise we consider the power spectrum of the signal $\mu(x)$ which is essentially proportional to $k^{-2 \alpha}$; we also consider the power spectrum of the condensate $\mathcal{O}(x)$ which we find numerically well approximated by $k^{-2 \Delta}$. We find that $\Delta \simeq 1.9+1.0 \alpha$ is therefore larger than $\alpha$ signaling that the weight of the high- $k$ harmonics is smaller in $\mathcal{O}$ than in $\mu$. Similarly, we approximate the power spectrum of the charge density $\rho(x)$ as $k^{-2 \Gamma(\alpha)}$, but this time we find $\Gamma<\alpha$. Below we present a plot of $\Delta$ and $\Gamma$ versus $\alpha$ for a wide range of values. The error bars were computed based on considering many realizations of the given noise.

We have gathered evidence that this behavior is also rather independent of the value of the mass of the scalar field, or, in the field theory language, it is independent of the conformal dimension of the order parameter. For example, we have confirmed a similar behavior for $m^{2}=0$. This universality of renormalization group $(\mathrm{RG})$ is one of the main observations of our work and its origin seems to be in the strongly coupled nature of the problem. The weak field theory intuition would dictate that $\Delta$ should be well approximated by the conformal dimension associated with the order parameter and here we verify that it is not.

It is also interesting to point out that this behavior does not depend on any of the parameters of our theory, i.e. $k_{0}$, $\mu_{0}$ or $\epsilon$. This means that we can redo Fig. 5 for the charge density in the normal phase. This particular case is interesting, since the theory becomes linear and we can therefore separate variables. Being that the case, we can recompute the power spectrum solving the equations of motion using a simple MATHEMATICA NDSolve command and we get $\Gamma=-1.0+1.0 \alpha$. The slope of this fit agrees with that found both for the condensate and the charge
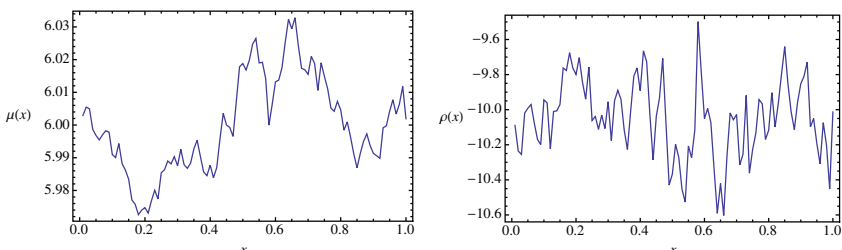

FIG. 4 (color online). Initial chemical potential profile $\mu(x)=$ $6.0+0.1 \sum_{n=1}^{100} \frac{1}{2 \pi n} \cos \left(2 \pi n x+\delta_{n}\right)$ (left panel) and the corresponding charge density profile (right panel). 
HOLOGRAPHIC SUPERCONDUCTOR WITH DISORDER
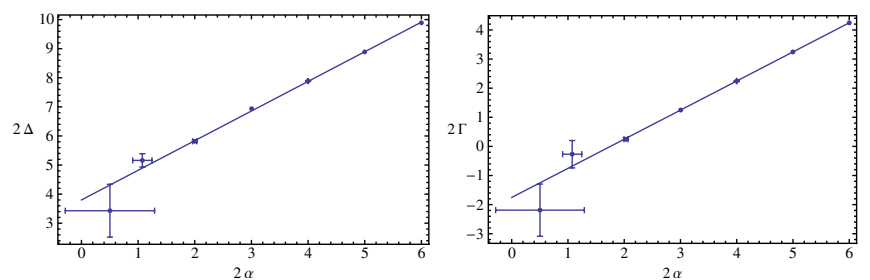

FIG. 5 (color online). Renormalization of the disorder: Condensate $\Delta=1.9+1.0 \alpha$ (left panel) and charge density $\Gamma=-0.88+1.0 \alpha$ (right panel). This plot was made considering $k_{0}=2 \pi, \mu_{0}=6$ and $\epsilon=1$. We can see that the flatter the spectrum, the larger the error. This might be associated with the fact that large momenta are more sensitive to the discretization.

density in the broken phase (Fig. 5) [27]. As for the ordinate we expect the apparent discrepancy to vanish for numerics with thinner grids.

\section{CONCLUSIONS}

In this paper we report two interesting findings: (i) The critical temperature of holographic superconductors increases with the increase in the strength of disorder and (ii) the power spectrum of the condensate and charge density are governed by fairly universal relations depending on the power spectrum of the original random signal.

Let us cautiously compare our results with the situation in the condensed matter literature. In the condensed matter literature about superconductors some results point to a degrading of $T_{c}$ with the strength of the disorder [17,26]. Other results, however, point to an enhancement of $T_{c}$ [28]. The precise role of the interactions in these studies is hard to gauge. Our results clearly point to an enhancement of $T_{c}$ but we should warn the reader that the role of interactions in our context is central and direct comparison with previous studies in the condensed matter literature will require a considerable amount of work likely at the level of [2] for dirty superconductors, that is, an analysis able to sum the electron-electron perturbation theory to all orders.

It might be more pertinent to compare our results with the literature for strongly coupled disordered superfluids. Indeed the numerical simulations of $[23,24]$ have shown that disorder can trigger an insulator to superfluid phase transition in systems that can be of relevance both for superfluids and high- $T_{c}$ superconductors.
It is worth pointing out that other holographic discussions, which could be considered as technical precursors to our work in that they solved simplified versions of our system, seem to also point to an enhancement of $T_{c}$. For example, [29] considered a time-dependent stimulation that lead, for a range of frequencies to increasing $T_{c}$, see however [30] (also [31]). Spatially modulated chemical potentials considered, for example, in [32] and [33] (see also [34]) point instead to a reduction of $T_{c}$.

The universality of the result $k^{-\alpha_{\mapsto}} \rightarrow\left(k^{-\Delta(\alpha)}, k^{-\Gamma(\alpha)}\right)$ for the order parameter and the charge density seems to be a general property of the gravity equations of motion. We have also provided supporting evidence that this result is largely independent of the value of the mass of the scalar field. An immediate and intriguing conclusion is that the operator mostly responsible for the RG is not necessarily the one to which the scalar field couples. This "universality" is interesting in the framework of the AdS/CFT and deserves further investigation.

The enhancement of $T_{c}$ with the strength of the noise in holographic superconductors deserves more scrutiny. It could be an important prediction of holographic superconductivity. It would be particularly interesting to consider other types of holographic superconductors (like, for instance, $p$ wave [35]), and carry out a similar analysis there. We leave this for future investigation.

In this manuscript we focused largely on the behavior of the condensate and the charge density averaged over the $x$ direction. There seems to be a rich structure in the $x$ dependence of such quantities (see Figs. 3 and 4). In particular, the condensate seems to show potential islands of superconductivity. It would be interesting to pursue the appearance of islands of superconductivity and the effect of different noises on the conductivity (see [36]). We hope to address such questions in a separate publication.

\section{ACKOWLEDGMENTS}

We are thankful to C. Herzog, J. Maldacena, D. Musso, J. Sonner, D. Tielas, D. Vaman, and C. Wu. D. A. and L.P.Z. acknowledge hospitality of the MCTP and ICTP, respectively. L. P. Z. and A. S. acknowledge the hospitality of GGI during the gestation of the project. D. A. thanks the FRoGS for unconditional support. This work was supported by the DoE Grant No. DE-SC0007859.
[1] P. W. Anderson, Phys. Rev. 109, 1492 (1958).

[2] D. Basko, I. Aleiner, and B. Altshuler, Ann. Phys. (Amsterdam) 321, 1126 (2006).

[3] V. Oganesyan and D. A. Huse, Phys. Rev. B 75, 155111 (2007).
[4] A. Pal and D. Huse, Phys. Rev. B 82, 174411 (2010).

[5] Shankar Iyer, Vadim Oganesyan, Gil Refael, and David A. Huse, Phys. Rev. B 87, 134202 (2013).

[6] A. De Luca and A. Scardicchio, Europhys. Lett. 101, 37003 (2013). 
D. AREÁN et al.

[7] F. Buccheri, A. De Luca, and A. Scardicchio, Phys. Rev. B 84, 094203 (2011).

[8] S. A. Hartnoll, P. K. Kovtun, M. Muller, and S. Sachdev, Phys. Rev. B 76, 144502 (2007).

[9] S. A. Hartnoll and C. P. Herzog, Phys. Rev. D 77, 106009 (2008).

[10] M. Fujita, Y. Hikida, S. Ryu, and T. Takayanagi, J. High Energy Phys. 12 (2008) 065.

[11] S. Ryu, T. Takayanagi, and T. Ugajin, J. High Energy Phys. 04 (2011) 115.

[12] A. Adams and S. Yaida, arXiv:1102.2892.

[13] A. Adams and S. Yaida, arXiv:1201.6366.

[14] O. Saremi, arXiv:1206.1856.

[15] P. Anderson, J. Phys. Chem. Solids 11, 26 (1959).

[16] S. Maekawa and H. Fukuyama, J. Phys. Soc. Jpn. 51, 1380 (1982).

[17] A. Kapitulnik and G. Kotliar, Phys. Rev. Lett. 54, 473 (1985); G. Kotliar and A. Kapitulnik, Phys. Rev. B 33, 3146 (1986).

[18] M. Ma and P. A. Lee, Phys. Rev. B 32, 5658 (1985).

[19] S. A. Hartnoll, C. P. Herzog, and G. T. Horowitz, Phys. Rev. Lett. 101, 031601 (2008).

[20] S. A. Hartnoll, C. P. Herzog, and G. T. Horowitz, J. High Energy Phys. 12 (2008) 015.

[21] S. A. Hartnoll, Classical Quantum Gravity 26, 224002 (2009).

[22] G. T. Horowitz, Lect. Notes Phys. 828, 313 (2011).

[23] L. Dang, M. Boninsegni, and L. Pollet, Phys. Rev. B 79, 214529 (2009).
PHYSICAL REVIEW D 89, 106003 (2014)

[24] J. W. Lee, S. Chandrasekharan, and H. U. Baranger, arXiv: cond-mat/0611109.

[25] P. Phillips, Advanced Solid State Physics (Cambridge University Press, Cambridge, 2012).

[26] M. Chand, G. Saraswat, A. Kamlapure, M. Mondal, S. Kumar, J. Jesudasan, V. Bagwe, L. Benfatto, V. Tripathi, and P. Raychaudhuri, Phys. Rev. B 85, 014508 (2012).

[27] A similar behavior was observed in [37] for the spectrum of a scalar operator.

[28] D. Fisher, M. P. Fisher, and D. Huse, Phys. Rev. B 43, 130 (1991).

[29] N. Bao, X. Dong, E. Silverstein, and G. Torroba, J. High Energy Phys. 10 (2011) 123.

[30] M. Natsuume and T. Okamura, J. High Energy Phys. 08 (2013) 139.

[31] W.-J. Li, Y. Tian, and H. Zhang, J. High Energy Phys. 07 (2013) 030.

[32] R. Flauger, E. Pajer, and S. Papanikolaou, Phys. Rev. D 83, 064009 (2011).

[33] J. Erdmenger, X.-H. Ge, and D.-W. Pang, J. High Energy Phys. 11 (2013) 027.

[34] S. K. Domokos, C. Hoyos, and J. Sonnenschein, J. High Energy Phys. 11 (2013) 172.

[35] S. S. Gubser and S. S. Pufu, J. High Energy Phys. 11 (2008) 033.

[36] S. A. Hartnoll and D. M. Hofman, Phys. Rev. Lett. 108, 241601 (2012).

[37] S. A. Hartnoll and J. E. Santos, arXiv:1402.0872. 